\title{
Front Matter: Volume 10540
}

, "Front Matter: Volume 10540," Proc. SPIE 10540, Quantum Sensing and Nano Electronics and Photonics XV, 1054001 (29 June 2018); doi: 10.1117/12.2319063

SPIE. Event: SPIE OPTO, 2018, San Francisco, California, United States 


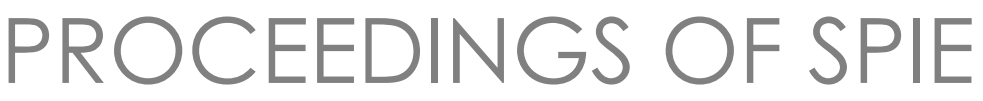

\title{
Quantum Sensing and Nano Electronics and Photonics XV
}

\author{
Manijeh Razeghi \\ Gail J. Brown \\ Jay S. Lewis \\ Giuseppe Leo \\ Editors
}

28 January-2 February 2018

San Francisco, California, United States

Sponsored and Published by

SPIE 
The papers in this volume were part of the technical conference cited on the cover and title page. Papers were selected and subject to review by the editors and conference program committee. Some conference presentations may not be available for publication. Additional papers and presentation recordings may be available online in the SPIE Digital Library at SPIEDigitallibrary.org.

The papers reflect the work and thoughts of the authors and are published herein as submitted. The publisher is not responsible for the validity of the information or for any outcomes resulting from reliance thereon.

Please use the following format to cite material from these proceedings:

Author(s), "Title of Paper," in Quantum Sensing and Nano Electronics and Photonics XV, edited by Manijeh Razeghi, Gail J. Brown, Jay S. Lewis, Giuseppe Leo, Proceedings of SPIE Vol. 10540 (SPIE, Bellingham, WA, 2018) Seven-digit Article CID Number.

ISSN: 0277-786X

ISSN: 1996-756X (electronic)

ISBN: 9781510615656

ISBN: 9781510615663 (electronic)

Published by

SPIE

P.O. Box 10, Bellingham, Washington 98227-0010 USA

Telephone +1360 676 3290 (Pacific Time) · Fax +1 3606471445

SPIE.org

Copyright @ 2018, Society of Photo-Optical Instrumentation Engineers.

Copying of material in this book for internal or personal use, or for the internal or personal use of specific clients, beyond the fair use provisions granted by the U.S. Copyright Law is authorized by SPIE subject to payment of copying fees. The Transactional Reporting Service base fee for this volume is $\$ 18.00$ per article (or portion thereof), which should be paid directly to the Copyright Clearance Center (CCC), 222 Rosewood Drive, Danvers, MA 01923. Payment may also be made electronically through CCC Online at copyright.com. Other copying for republication, resale, advertising or promotion, or any form of systematic or multiple reproduction of any material in this book is prohibited except with permission in writing from the publisher. The CCC fee code is 0277 $786 \mathrm{X} / 18 / \$ 18.00$.

Printed in the United States of America.

Publication of record for individual papers is online in the SPIE Digital Library.

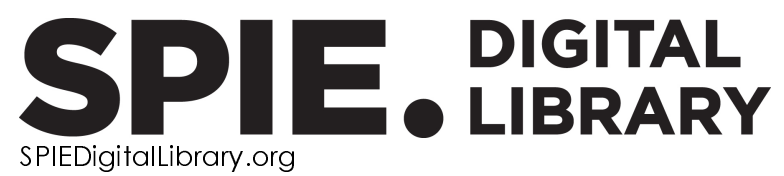

Paper Numbering: Proceedings of SPIE follow an e-First publication model. A unique citation identifier (CID) number is assigned to each article at the time of publication. Utilization of CIDs allows articles to be fully citable as soon as they are published online, and connects the same identifier to all online and print versions of the publication. SPIE uses a seven-digit CID article numbering system structured as follows:

- The first five digits correspond to the SPIE volume number.

- The last two digits indicate publication order within the volume using a Base 36 numbering system employing both numerals and letters. These two-number sets start with $00,01,02,03,04$, 05, 06, 07, 08, 09, 0A, OB ... 0Z, followed by 10-1Z, 20-2Z, etc. The CID Number appears on each page of the manuscript. 


\title{
Contents
}

\author{
vii Authors \\ xi Conference Committee
}

\section{KEYNOTE SESSION}

1054002 Application of IR thermometry to understanding brain function (Keynote Paper) [10540-1]

1054004 Type-II InAs/GaSb/AISb superlattice-based heterojunction phototransistors: back to the future (Keynote Paper) [10540-3]

\section{ADVANCED SENSING TECHNOLOGY}

1054005 Understanding fundamental limitations of materials to enable advanced design (Keynote Paper) [10540-4]

1054007 Double electron barrier structure for suppression of dark current in microjunction-based type-II InAs/InAsSb superlattice long-wavelength infrared photodetectors [10540-6]

\section{QUANTUM CASCADE LASERS I}

1054009 Mid-infrared interband cascade light-emitting devices with improved radiance (Invited Paper) [10540-8]

10540 OA Broadband monolithically tunable quantum cascade lasers (Invited Paper) [10540-9]

$10540 \mathrm{OB}$ Gain modeling and thermal management of optically pumped semiconductor lasers (Invited Paper) [10540-10]

10540 OC Interband cascade laser based quartz-enhanced photoacoustic sensor for multiple hydrocarbons detection [10540-11]

\section{DETECTORS AND SENSORS}

10540 OE Gain and resonant tunneling in interband cascade IR photodetectors (Invited Paper) [10540-13]

$10540 \mathrm{OF}$ Intersubband polaritons are robust against inhomogeneous broadening induced by multiple quantum wells (Invited Paper) [10540-14] 
10540 OG A minimized ultra-sensitive MIR hollow waveguide (HWG) isotope ratio analyzer for environmental and industrial applications (Invited Paper) [10540-15]

$10540 \mathrm{Ol} \quad$ Sofradir vertical industrial model for high-image-quality MCT detectors [10540-17]

IMAGING SCIENCE AND TECHNOLOGY I

10540 OL Development of an InGaAs SPAD 2D array for flash LIDAR (Keynote Paper) [10540-19]

10540 ON Sub-wavelength near field imaging techniques at terahertz frequencies (Invited Paper) [10540-21]

IMAGING SCIENCE AND TECHNOLOGY II

10540 OP Extended short wavelength infrared FPA technology: status and trends (Invited Paper) [10540-23]

10540 OQ Development technology of principle prototype of high-resolution quantum remote sensing imaging (Invited Paper) [10540-24]

PLASMONICS AND 2D SYSTEMS

10540 OS Photovoltages and hot electrons in plasmonic nanogaps (Keynote Paper) [10540-26]

EMERGING PHOTONIC DEVICES I

10540 OX Highly stable forced optoelectronic oscillators and roadmap to integrated clocks (Keynote Paper) [10540-32]

$10540 \mathrm{OZ}$ One quantum sensor for all gases: cavity-enhanced Raman spectroscopy for food-chain monitoring (Invited Paper) [10540-34]

1054011 Ge-rich SiGe photonic-integrated circuits for mid-IR spectroscopy (Invited Paper) [10540-36]

\section{EMERGING PHOTONIC DEVICES II}

1054012 Directed emission by electrically driven optical antennas (Invited Paper) [10540-37]

1054016 Incident femtosecond pulse chirp influence on nonlinear localization of laser energy in 2D photonic crystal (Invited Paper) [10540-42] 
10540 1D Microcavity III-V lasers monolithically grown on silicon (Invited Paper) [10540-48]

$105401 \mathrm{E}$ Optical quality in strain-balanced InAs/InAsSb superlattices grown with and without Bi surfactant (Invited Paper) [10540-49]

$105401 G \quad$ Novel cubic phase III-nitride complementary metal-oxide-semiconductor transistor technology (Invited Paper) [10540-51]

$105401 \mathrm{H} \quad$ The Kerr phase-interrogator: exploiting the nonlinear Kerr-effect for overcoming fundamental limitations in linear sensing approaches (Invited Paper) [10540-52]

\section{QUANTUM CASCADE LASERS II}

$1054011 \quad$ Controlling QCLs for frequency metrology from the infrared to the THz range (Keynote Paper) [10540-53]

$105401 \mathrm{~J}$ Development of THz light sources based on QCL technology (Invited Paper) [10540-54]

10540 IM Talbot coupling of an array of quantum cascade lasers (Invited Paper) [10540-57]

$105401 \mathrm{~N}$ Temperature dependence of a mid-infrared quantum cascade laser with external optical feedback (Invited Paper) [10540-58]

\section{QUANTUM CASCADE LASERS III}

1054010 Compact and low power-consumption MIR DFB-QCL with To-CAN package for portable sensor (Invited Paper) [10540-59]

10540 1P Progress on the transistor-injected quantum-cascade laser (Invited Paper) [10540-60]

$105401 Q \quad$ Compact and low-noise quartz-enhanced photoacoustic sensor for sub-ppm ethylene detection in atmosphere (Invited Paper) [10540-61]

\section{ADVANCES IN LASER TECHNOLOGY}

10540 is Bases and experimental validation of a novel VSPIN model: towards functional spincontrolled VCSELs (Keynote Paper) [10540-63]

$105401 \mathrm{~V}$ Polariton-lasing in microcavities filled with fluorescent proteins (Invited Paper) [10540-66]

$105401 \mathrm{~W}$ Super-resonant coherent absorption sensing (Invited Paper) [10540-67] 
$105401 \mathrm{X}$ Terahertz detection in 2D materials (Invited Paper) [10540-68]

1054020 Resonant scattering probes for terahertz near-field microscopy (Invited Paper) [10540-72]

FREQUENCY COMBS AND NONLINEAR PROCESSES II

10540 2B Nonlinear dynamics in multimode optical fibers (Invited Paper) [10540-79]

$105402 \mathrm{D}$ Comb generation of second and third harmonics at high-intensive femtosecond pulse in medium with quadratic and cubic nonlinearities (Invited Paper) [10540-82]

$105402 \mathrm{E} \quad$ Results of very long time ageing tests of DFB ridge laser diodes emitting at $852 \mathbf{n m}$ and 894 nm for Cesium atomic clocks (Invited Paper) [10540-83]

INFRARED DETECTORS I

$105402 \mathrm{G}$ SPAD-based flash LiDAR sensor with high ambient light rejection for automotive applications (Invited Paper) [10540-85]

1054021 Compact low-power mid-IR supercontinuum for sensing applications (Invited Paper) [10540-88]

INFRARED DETECTORS II

$105402 \mathrm{~K}$ Remote detection of leakages of non-IR-active gases by laser spectroscopy (Invited Paper) [10540-89]

$105402 \mathrm{~L} \quad$ Tapered hollow-core fibers providing single-mode output in the $3.5 \mu \mathrm{m}-7.8 \mu \mathrm{m}$ spectral range [10540-90]

1054020 Recent advances in quartz-enhanced photoacoustic sensing (Invited Paper) [10540-92] 


\section{Authors}

Numbers in the index correspond to the last two digits of the seven-digit citation identifier (CID) article numbering system used in Proceedings of SPIE. The first five digits reflect the volume number. Base 36 numbering is employed for the last two digits and indicates the order of articles within the volume. Numbers start with 00, 01, 02, 03, 04, 05, 06, 07, 08, 09, OA, OB...0Z, followed by 10-12, 20-2Z, etc.

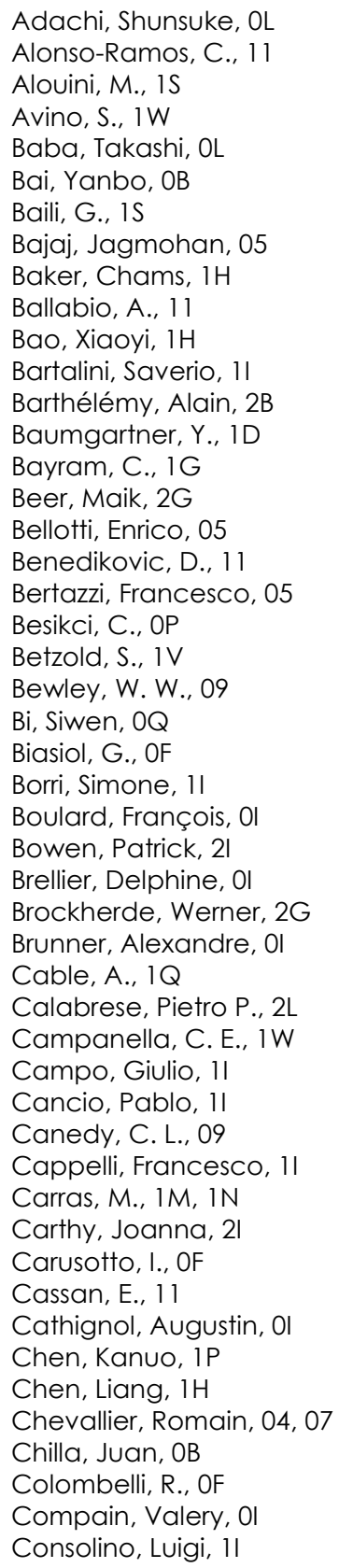

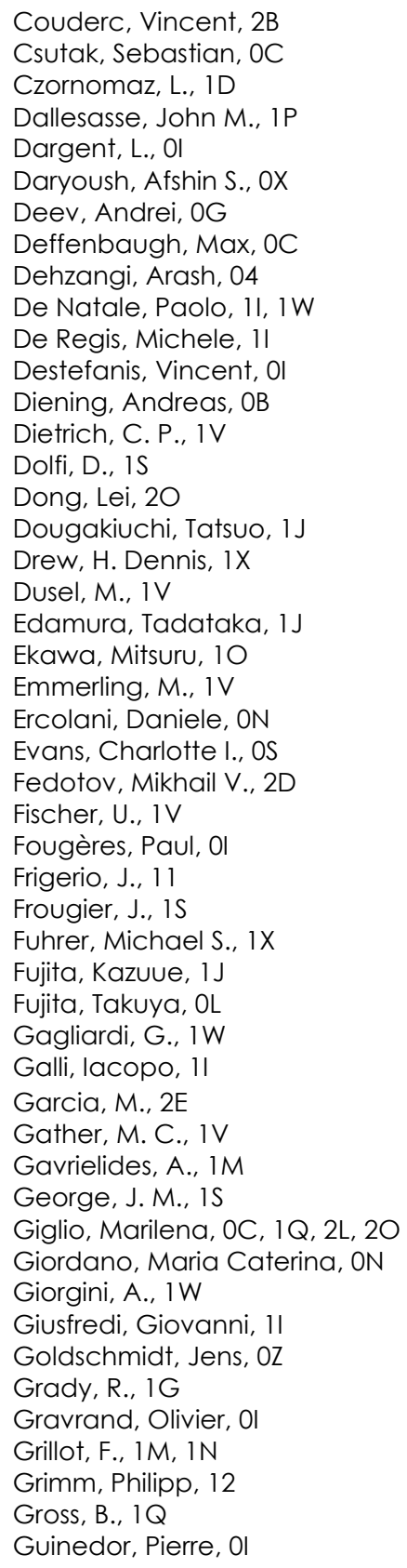


Haase, Jan F., 2G

Haddadi, Abbas, 04, 07

Hashi, Tatsuya, OL

Hashimoto, Jun-ichi, 10

Hecht, Bert, 12

Herbst, Johannes, 2K

Hillenbrand, Rainer, ON

Hitaka, Masahiro, $1 \mathrm{~J}$

Höfling, S., IV

Hooper, Lucy, 21

Hosticka, Bedrich J., $2 G$

Hsiao, Fu-Chen, $1 \mathrm{P}$

Huang, Wenxiang, $\mathrm{OE}$

Huot, Laurent, 2

Iguchi, Yasuhiro, 10

lorga, Michael, 02

Isella, G., 11

Ito, Akio, $1 \mathrm{~J}$

Jadidi, M. Mehdi, IX

Johnson, Shane R., $1 \mathrm{E}$

Joly, A., is

Kapferer, Armelle, 0 I

Kato, Takashi, 10

Katsuyama, Tsukuru, 10

Kerlain, Alexandre, 0

Khanal, S., $1 \mathrm{~N}$

Kharitonov, Dmitry M., 2D

Kim, C. S., 09

Kim, M., 09

Kokozinski, Rainer, 2G

Krakowski, M., 2E

Kriesel, Jason M., 2L

Kronenberg, N., IV

Krupa, Katarzyna, 2B

Kullock, René, 12

Lambrecht, Armin, 2K

Le Roux, X., 11

Lei, Lin, OE

Li, LU, OE

Lin, Xuling, $O Q$

Liv, Huiyun, 20

Liu, Q., 11

Loginova, Maria M., 16, 2D

Lysak, Tatiana M., 16

Mackowiak, V., $1 Q$

Maier, Eric, $2 \mathrm{~K}$

Makino, Kenji, OL

Malara, P., IW

Manceau, J-M., OF

Marris-Morini, D., 11

Massengale, Jeremy A., OE

Mastel, Stefan, ON

Mauthe, S., ID

Mayer, B., 1D

Mazzotti, Davide, II

McClintock, Ryan, OA

Menduni, Giansergio, OC

Merritt, C. D., 09

Meyer, J. R., 09

Migita, Masaki, 10
Millot, Guy, 2B

Mishima, Tetsuya D., OE

Mitrofanov, Oleg, 0N, 20

Mittendorff, Martin, IX

Modotto, Daniele, 2B

Mori, Hiroki, 10

Moselund, K. E., 1D

Moselund, Peter M., 21

Murata, Makoto, 10

Murphy, Thomas E., $1 \mathrm{X}$

Nakamura, Shigeyuki, OL

Natelson, Douglas, OS

Natrella, Michele, 20

Newell, T. C., $1 \mathrm{M}$

Ochs, Maximilian, 12

Ohmer, J., $1 \mathrm{~V}$

Palzer, Stefan, $\mathrm{OZ}$

Parillaud, O., 2E

Park, K., $1 \mathrm{G}$

Parrish, Todd, 02

Passaro, Vittorio, OC

Patimisco, Pietro, 0C, 1Q, 2L, 20

Perconti, Philip, 05

Powell, Ross, 21

Rademacher, Sven, 2K

Ramirez, J. M., 11

Razeghi, Manijeh, 04, 07, 0A

Reed, Meredith, 05

Resneau, P., 2E

Robert, Y., 2E

Rossmadl, H., 1Q

Rubaldo, Laurent, Ol

Ruskowski, Jennifer, 2G

Sam-Giao, D., Ol

Sampaolo, Angelo, 0C, 1Q, 2L, 20

Sandfort, Vincenz, $\mathrm{OZ}$

Santos, Michael B., OE

Scamarcio, Gaetano, ON

Schaefer, Stephen T., IE

Schmid, H., 1D

Scholz, Christian, OB

Schrey, Olaf M., 2G

Schubert, M., IV

Schuster, Jonathan, 05

Serna, S., 11

Sgobba, F., 1Q

Siday, Tom, 20

Slivken, Steven, OA

Sorba, Lucia, ON

Sousa, M., 1D

Spagnolo, Vincenzo, 0C, 1Q, 2L, 20

Spitz, O., 1M, 1N

Staudinger, P., 1D

Steenbergen, Elizabeth H., $1 \mathrm{E}$

Strahl, Thomas, 2K

Sushkov, Andrei B., $1 \mathrm{X}$

Suzuki, Yoshihito, OL

Tittel, Frank K., OC, 1Q, 2L, 20

Tonello, Alessandro, 2B

Tran, N. L., OF 
Trofimov, Vyacheslav A., 16, 2D

Tropf, L., IV

Trykin, Evgeny M., 16

Tsuji, Yukihiro, 10

Vakarin, V., 11

Vinet, E., 2E

Viti, Leonardo, ON

Vitiello, Miriam S., ON

Vivien, L., 11

Von Bandel, N., 2E

Vurgaftman, I., 09

Wabnitz, Stefan, 2B

Wang, Zhenyou, OG

Warren, M. V., 09

Webster, Preston T., $1 \mathrm{E}$

Williams, B. S., 1N

Winniger, J., ID

Wirths, S., ID

Wisdom, Jeffrey, OB

Wöllenstein, Jürgen, $0 Z$

Wong, C. W., IN

Wu, Donghai, OA

Wu, J., $1 \mathrm{~N}$

Wu, Jiang, 20

WU, Sheng, OG

WU, Zhiqiang, $O Q$

Yamamoto, Koei, OL

Yang, Rui Q., OE

Yang, Song, $O Q$

Yang, Thomas, 04

Yoshinaga, Hiroyuki, 10

Zhou, Wenjia, OA

Zhuang, Yan, OG

Zolotavin, Pavlo, OS

Proc. of SPIE Vol. $105401054001-9$ 
Proc. of SPIE Vol. 10540 1054001-10 Downloaded From: https://www.spiedigitallibrary.org/conference-proceedings-of-spie on 26 Apr 2023
Terms of Use: https://www.spiedigitallibrary.org/terms-of-use 


\title{
Conference Committee
}

\author{
Symposium Chairs
}

Connie J. Chang-Hasnain, University of California, Berkeley (United States)

Graham T. Reed, Optoelectronics Research Centre, University of Southampton (United Kingdom)

Symposium Co-chairs

Jean-Emmanuel Broquin, IMEP-LAHC (France)

Shibin Jiang, AdValue Photonics, Inc. (United States)

Program Track Chairs

Ali Adibi, Georgia Institute of Technology (United States)

David L. Andrews, University of East Anglia (United Kingdom)

Conference Chair

Manijeh Razeghi, Northwestern University (United States)

Conference Co-chairs

Gail J. Brown, Air Force Research Laboratory (United States)

Jay S. Lewis, Defense Advanced Research Projects Agency (United States)

Giuseppe Leo, Université Paris Diderot (France)

Conference Program Committee

Steven B. Brown, Space Dynamics Laboratory (United States)

David A. Cardimona, Air Force Research Laboratory (United States)

Vincent M. Cowan, Air Force Research Laboratory (United States)

Paolo De Natale, Istituto Nazionale di Ottica (Italy)

Jérôme Faist, ETH Zürich (Switzerland)

Michael D. Gerhold, U.S. Army Research Office (United States)

Robert J. Grasso, EOIR Technologies (United States)

Frédéric Grillot, Télécom ParisTech (France)

Yasar Gurbuz, Sabanci University (Turkey)

Sven Höfling, University of St. Andrews (United Kingdom)

John E. Hubbs, Ball Aerospace \& Technologies Corporation

(United States)

Jean-Pierre Huignard, Jphopto (France) 
Woo-Gwang Jung, Kookmin University (Korea, Republic of)

Tsukuru Katsuyama, Sumitomo Electric Industries, Ltd. (Japan)

Giti A. Khodaparast, Virginia Polytechnic Institute and State University (United States)

Michel Krakowski, Thales Research \& Technology (France)

Kwok Keung Law, Naval Air Warfare Center Weapons Divison (United States)

Craig M. Lennon, U.S. Army Night Vision \& Electronic Sensors

Directorate (United States)

Amy W. K. Liu, IQE Inc. (United States)

Jerry R. Meyer, U.S. Naval Research Laboratory (United States)

Maya Mikhaliova, loffe Physico-Technical Institute (Russian Federation)

Jan Misiewicz, Wroclaw University of Technology (Poland)

Oleg Mitrofanov, University College London (United Kingdom)

Shanee Pacley, Air Force Research Laboratory (United States)

Dimitris Pavlidis, Boston University (United States)

Jean-Luc Pelouard, Joint Research Laboratory of Micro and Nano Optics (MiNaO) (France)

Narasimha S. Prasad, NASA Langley Research Center (United States)

Edward H. Sargent, University of Toronto (Canada)

Gaetano Scamarcio, Università degli Studi di Bari Aldo Moro (Italy)

Elizabeth H. Steenbergen, Air Force Research Laboratory (United States)

Marija Strojnik Scholl, Centro de Investigaciones en Óptica, A.C. (Mexico)

Meimei Tidrow, U.S. Army Night Vision \& Electronic Sensors Directorate (United States)

Cunzhu Tong, Changchun Institute of Optics, Fine Mechanics and Physics (China)

Luisa Torsi, Università degli Studi di Bari Aldo Moro (Italy)

Miriam Serena Vitiello, Consiglio Nazionale delle Ricerche (Italy)

\section{Session Chairs}

1 Keynote Session

Manijeh Razeghi, Northwestern University (United States)

Jerry R. Meyer, U.S. Naval Research Laboratory (United States)

2 Advanced Sensing Technology

Guanxi Andy Chen, The Boeing Company (United States)

Giuseppe Leo, Université Paris Diderot (France)

3 Quantum Cascade Lasers I

Todd B. Parrish, Northwestern University (United States)

Toshihiko Baba, Yokohama National University (Japan) 
Detectors and Sensors

Cengiz Besikci, Middle East Technical University (Turkey)

5 Imaging Science and Technology I

Manijeh Razeghi, Northwestern University (United States)

Giti A. Khodaparast, Virginia Polytechnic Institute and State University (United States)

6 Imaging Science and Technology II

Henri-Jean Drouhin, Ecole Polytechnique (France)

Venkatesh Narayanamurti, Harvard University (United States)

7 Plasmonics and 2D Systems

Ivano Alessandri, Università degli Studi di Brescia (Italy)

Giti A. Khodaparast, Virginia Polytechnic Institute and State University (United States)

8 Emerging Photonic Devices I

Giuseppe Leo, Université Paris 7-Diderot (France)

Jean-Luc Pelouard, Joint Research Laboratory of Micro and Nano Optics (MiNaO) (France)

9 Emerging Photonic Devices II

Mehdi Alouini, Institut FOTON (France)

Claire Deeb, C2N-CNRS (France)

10 Nanophotonics

Peter R. Herman, University of Toronto (Canada)

Can Bayram, University of Illinois (United States)

11 Optoelectronic Devices

Alexandre Bouhelier, Université de Bourgogne (France)

René Kullock, Julius-Maximilians-Universität Würzburg (Germany)

12 Quantum Cascade Lasers II

John Dallesasse, University of Illinois (United States)

Kirsten Moselund, IBM Research - Zürich (Switzerland)

13 Quantum Cascade Lasers III

Sven Höfling, Julius-Maximilians-Universität Würzburg (Germany)

Patrick Kung, The University of Alabama (United States)

14 Advances in Laser Technology

Cengiz Besikci, Middle East Technical University (Turkey)

Kamil Kosiel, Institute of Electron Technology (Poland) 
15 Terahertz and Nonlinear Spectroscopy Jérôme Faist, ETH Zurich (Switzerland)

Makoto Murata, Sumitomo Electric Industries, Ltd. (Japan)

16 Frequency Combs and Nonlinear Processes I

Afshin S. Daryoush, Drexel University (United States)

Steven B. Brown, Space Dynamics Laboratory (United States)

17 Frequency Combs and Nonlinear Processes II

Carlo Sirtori, Université Paris Diderot (France)

Sarah Houver, Laboratoire Pierre Aigrain (France)

18 Infrared Detectors I

Sylvain Combrié, Thales Research \& Technology (France)

19 Infrared Detectors II

Alexander Soibel, Jet Propulsion Laboratory (United States)

Henri-Jean Drouhin, Ecole Polytechnique (France) 